\title{
Overcoming Challenges in Collaboration between Research and Practice: The Agile Research Network
}

\author{
Helen Sharp and Laura Plonka \\ The Open University \\ Milton Keynes, Herts \\ MK7 6AA, UK \\ +44 1908653638 \\ (helen.sharp or laura.plonka)@open.ac.uk
}

\author{
Katie Taylor and Peggy Gregory \\ University of Central Lancashire \\ Preston, Lancs \\ PR1 2HE, UK \\ +44 1772 89(3321 or 3284) \\ (kjtaylor or ajgregory)@uclan.ac.uk
}

\begin{abstract}
There is wide acceptance in the software engineering field that industry and research can gain significantly from each other and there have been several initiatives for encouraging collaboration between the two. However there are some often-quoted challenges in this kind of collaboration. For example, that the timescales of research and practice are incompatible, that research is not seen as relevant for practice, and that research demands a different kind of rigour than practice supports. These are complex challenges that are not always easy to overcome. For the last year we have been using an approach designed to address some of these challenges and to bridge the gap between research and practice, specifically in the agile software development arena. So far we have collaborated successfully with two partners and have investigated two practitioner-driven challenges with agile. In this short paper we will introduce the approach, how it addresses the collaboration challenges between research and practice, and describe the lessons learned from our experience.
\end{abstract}

\section{Categories and Subject Descriptors}

D.2.9 [Software Engineering]: Management

\section{General Terms}

Human Factors

\section{Keywords}

Agile, Action Research, Project Management, Collaboration

\section{INTRODUCTION}

The Agile Research Network was set up in 2013 as a result of collaboration between the authors' institutions and an industry body, the DSDM Consortium [1]. It aims to bridge the gap between academia and industry in the field of Agile software development by providing a model for the delivery of timely and relevant research of use to practitioners.

Agile Software Development evolved during the 1990's as a response by 'grass root' developers to counter some of the known problems of the Waterfall lifecycle [2]. Agile methodologies

Permission to make digital or hard copies of all or part of this work for personal or classroom use is granted without fee provided that copies are not made or distributed for profit or commercial advantage and that copies bear this notice and the full citation on the first page. Copyrights for components of this work owned by others than ACM must be honored. Abstracting with credit is permitted. To copy otherwise, or republish, to post on servers or to redistribute to lists, requires prior specific permission and/or a fee. Request permissions from Permissions@acm.org.

SER\&IPs'14, June 1, 2014, Hyderabad, India

Copyright 2014 ACM 978-1-4503-2859-3/14/06...\$15.00

http://dx.doi.org/10.1145/2593850.2593859 typically use iterative and incremental development; advocate extensive business and user involvement; and use small crossfunctional teams to deliver a fit-for-purpose product on-time. Agile methods have become more main stream as organisations seek to scale-up the benefits it claims. However, the impact this way of working has on project teams and the wider organisation is less well understood. Research that gains greater understanding of how Agile methods work in industrial and commercial situations is required to assist organisational uptake.

Collaboration between individual researchers and practitioners is relatively unproblematic and not uncommon, especially in recent years. While this can yield solid benefits for the researcher, and useful insights for the practitioner involved, this approach has limitations due to issues of time, priorities and authority. Although a practitioner or group of practitioners may be motivated to engage in research, their time and priorities will be geared towards doing their job. Effort expended on supporting research is an 'overhead', and in addition, individuals' authority may be limited.

Greater benefits can be achieved for both sides by involving organisations more formally, and this inevitably involves funding and more careful scheduling. This leads to a different set of tensions, where agendas for the research may be directed by the partner providing the funding. If practice provides the funding, then the research agenda is largely determined by practitioners, and when research bodies provide the funding, the agenda is largely determined by researchers. Government schemes such as the TSB (Technology Strategy Board) in the UK have been successful in bringing partners together and providing some support for joint working, but in some cases, the situation calls for a partnership that is more responsive to practitioners' needs.

Over the past few years we have been trying to find a model of collaboration and funding that supports the agendas of individual organisations and the research agendas of individual research teams, and also allows for appropriate funding and authority. From our own experiences and those of others expressed at conference panels, and researcher and practitioner meetings, some of the key challenges in this kind of collaboration are:

Timeliness is a key issue for industry. The rigour highly prized by academia has a high cost in terms of time. Practitioners often see research as a long-winded process which will demand time from them but not necessarily provide significant benefit. Research outputs can take many months or sometimes years and from the practitioner's perspective the original issues will have changed, evolved or been superseded. Decisions will have been made based on the information available at the time. Practitioners are also wary of how much of their time this will take - as with software 
development customers, they want to tell us the problem and for us to go away and find a solution. Traditional research approaches require time to gather and analyse data in a rigorous fashion rather than answering today's industry-based problems.

Relevance is defined as addressing the needs of one or more different stakeholders [3]. Understanding the motivations of the different stakeholders is vital to ensure outputs are relevant. Relationship building is essential in order for academics to work closely with practitioners to understand context and business realities resulting in outputs that meet the needs of both stakeholder groups.

Industry seeks solutions to specific problems in a specific context. Academics, more often are concerned with generalisations and theory building. Research outputs need to be crafted in different ways to be relevant to different stakeholder groups. Typical academic outputs focus on positioning the work within an existing field, arguing for the suitability of research approach, and providing quality analysis and interpretation of data. Practitioners seek palatable solutions to specific problems or seek to learn about new tools or techniques. This is evidenced by the content of many practitioner conferences that contain experience reports with anecdotal focus on tools, practices or techniques of adoption.

Rigour: Quality research outputs require rigorous investigations, which are not necessarily compatible with commercial pressures. Practitioners will always be pragmatic at overcoming any challenges they face, and although solutions to a problem may be found, it is rare for thorough evaluation of any solutions or changes to take place.

Accessible: Academic research is usually written for an academic audience and is often written in a format that's not appropriate for practitioners Despite there being a wealth of knowledge available, few practitioners have the time, inclination or access to delve into existing research on a particular topic.
In the next section we introduce the ARN, in section 3 we describe examples from our two case studies so far, in section 4 we describe the lessons learned and we end with some conclusions.

\section{AGILE RESEARCH NETWORK (ARN)}

The Agile Research Network (ARN, agileresearchnetwork.org) was set up in response to the issues described above as a collaboration between The Open University (OU) and University of Central Lancashire (UCLan). ARN is currently supported by the universities themselves and funded by the DSDM Consortium (dsdm.org). ARN seeks to work closely with agile practitioners in their place of work to understand the implications and influences agile methods have on organisations and individuals, to assess the scope and nature of their effectiveness and then disseminate findings to a wider audience. We differ from consultants because we have time to investigate, observe and understand the problem situation; we work cooperatively with our collaborators but they remain fully in charge of any changes they wish to make; we use a model to structure our intervention and document our process throughout; we are not answerable to management as a consultant is; we are not selling a particular methodology, and we have not held back from highlighting deficiencies in methods where we have found them.

To address each of the concerns listed in Section 1 we have designed and implemented the approach shown in Figure 1. The approach has four distinct phases: Collaboration kick-off, investigation, implementation and evaluation. It is timeboxed and during each phase we work closely with the organisation concerned. In addition, the organisation will have had some contact with the research team before the collaboration kick-off starts. Specifically, collaborators are identified through a call for challenges distributed to the DSDM mailing list. This means that organisations approach the ARN team rather than the other way around.

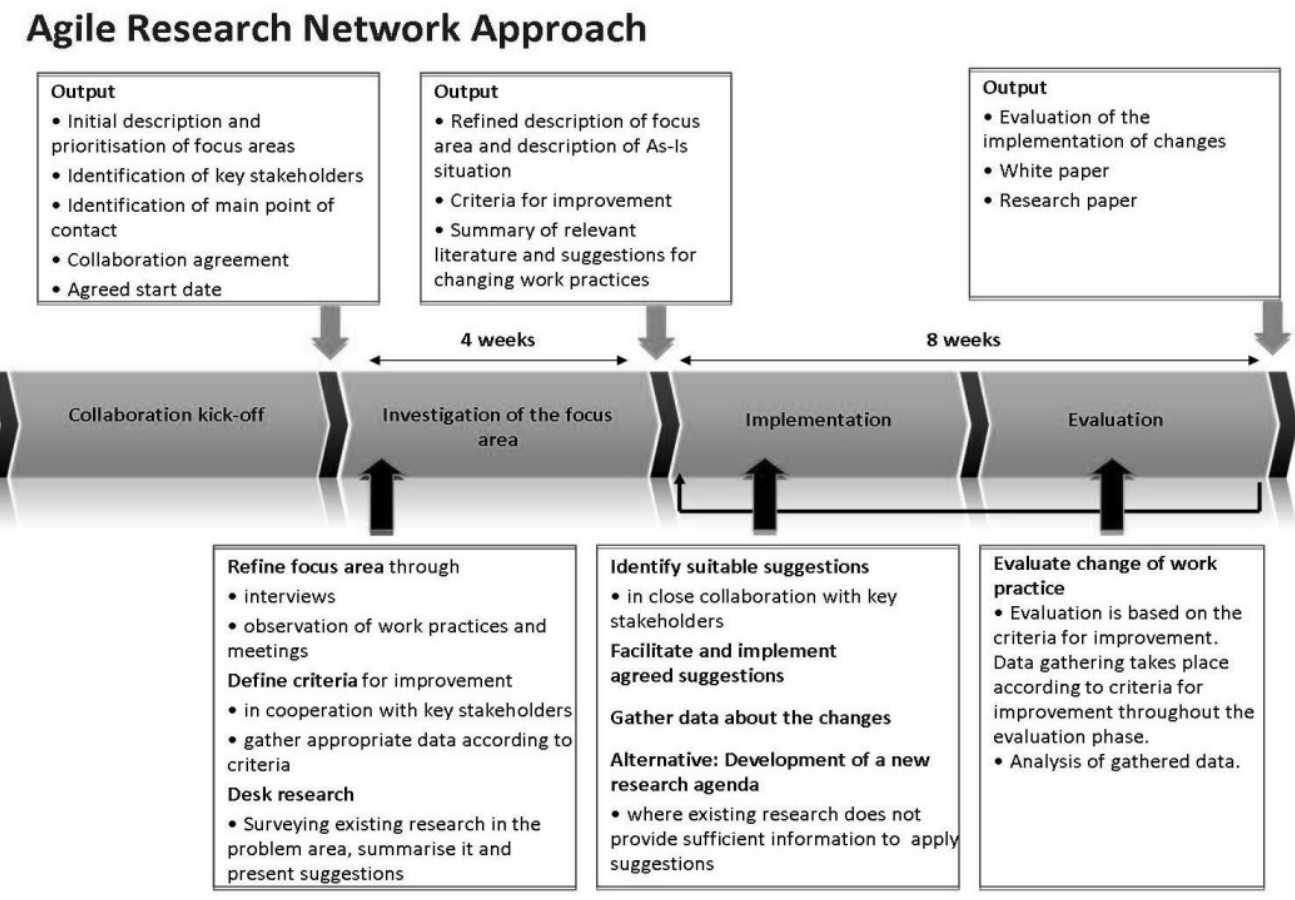

Figure 1: The Agile Research Network approach 
In Collaboration kick-off, the organisation and research team discuss the challenge and decide how best to proceed. The challenge is appropriate if:

- the researchers already have some understanding or expertise in the area

- literature exists specifically in the area or in a related area

- the challenge is significant enough to the organisation to maintain their engagement throughout the investigation

A gatekeeper for the organisation is identified, a collaboration agreement is drawn up and a start date agreed.

During Investigation, researchers spend time at the organisation scoping and defining the problem in detail. Different research methods may be applied to investigate the challenge and identify how improvements could be evaluated at the evaluation stage. Then relevant existing research and literature in the problem area is surveyed and summarised. This tailored literature review and specific suggestions are presented to the stakeholders.

At the Implementation stage, the organisation decides whether to adopt any of the suggestions presented. If they do, the research team facilitates the implementation of new ways of working. Alternatively, where existing research cannot provide suggestions or suggestions are not suitable for the context, a research agenda to investigate the challenge area in more detail can be developed.

During the evaluation phase, the research team evaluates the changes implemented at the organisation. The evaluation can include quantitative or qualitative measurements.

The ARN model addresses the concerns in Section 1 as follows:

Timeliness: The model uses short iterative research cycles. The timeframes shown on the model relate to 12 week elapsed time rather than calendar time as researchers are not full time and business priorities dictate access. If there is insufficient information on the challenge area and a new research agenda is developed a new timeline is set up.

Relevance: At the end of each research cycle we seek feedback from the organisation to ensure the challenges we are addressing are still relevant and that we have understood the context correctly. This constant feedback between researchers and stakeholders increases the rigour of our process and allows practitioners to assess and move forward in the areas where there is most value.

Accessible: As academics we have access to a wide range of research literature. We are able to search, synthesise and present this in a format that is useful to practitioners in specific contexts. We also ensure our outputs are accessible to a range of stakeholders. Each engagement produces a specific report and presentation for the organisation, a practitioner-focused whitepaper and an academic output.

\section{EXAMPLES FROM OUR WORK}

Up to now, the ARN approach has been applied in two case studies. The first case study was conducted in collaboration with a hi-tech software development company that approached the ARN with the challenge of integrating the UX design into a DSDM project. For the second case study the ARN worked with an organisation that faced the challenge of running agile projects and reporting agile progress in a non-agile environment.
Below, we present examples of how the ARN approach addressed the challenges of Timeliness, Relevance and Accessibility in each of these two case studies.

\subsection{First case study: Integrating UX design into a DSDM project}

Timeliness: The majority of the case study took place between April and October 2013. After all collaboration documents were agreed in April 2013, the investigation phase took place between May and July 2013. Based on the findings of the investigation phase the first major output for the company, the suggestions for improvement were presented at the end of July 2013 to the project team. The second major output for industry, the White Paper, was jointly written with the company during September/October and was presented and published in October 2013.

Despite the timely delivery of the industry focused outputs, we encountered some difficulties. For example, the investigation phase required some flexibility in terms of the researchers' time as the activities during that phase were driven by the availability of our point of contact and other key stakeholders, as well as the project priorities. The project focus varied from timebox to timebox and while in some timeboxes several UX design related activities took place, in other timeboxes the development team focused exclusively on the technical implementation. This meant that there were times in which our investigation did not progress as quickly as we had initially expected.

Relevance: During the investigation phase (May to July 2013), we regularly met informally with the managing director and project manager to discuss our observations and findings. These conversations allowed the company to reflect on their challenges early in the case study and supported us in producing rigorous research outputs by evaluating our observations. They also led to joint understanding of the challenge area and informed the next steps of our data gathering i.e. who to interview next, what kind of questions to ask to investigate the most relevant aspects of the challenge.

Accessible: In July 2013, at the end of the investigation phase, we set up a two hour meeting with the project team to discuss the challenges we had identified through the data gathering, and to suggest alternative ways of working based on existing literature. For this meeting, we reviewed a wide range of literature including papers published at conferences and in journals, books and websites. We identified applicable suggestions based on nine papers published at conferences, two journal papers, one book and two websites. Project leads from both case studies reported that the suggestions for improvements were very useful and were actively discussed by the project team.

\subsection{Second case study: Reporting agile progress in non-agile environments}

Timeliness: The first point of contact with the organisation was in May 2013 but the case study started in July 2013 after a suitable challenge was identified and the collaboration set up; this study is still ongoing. After a first investigation of the challenge area, we conducted interviews with different stakeholders in August 2013 and analysed the data in September/October 2013. We discussed the findings and remaining open questions with key stakeholders in November 2013 and presented the suggestions for improvements 
back to the organisation in December 2013. We are currently working on the White paper and planning to publish the White Paper in February/March 2014.

Relevance: Although we had one specific challenge area identified during the collaboration kick off, the interviews during the investigation phase revealed a range of different challenges and different perspectives. Towards the end of the investigation phase, we had to decide which challenges we should focus on when reviewing existing literature. We set up a meeting with our main point of contact to present the different challenge areas and to identify the most relevant areas for their organisation.

Accessible: In December 2013, we presented suggestions back to the organisation on four different challenge areas. Research was sparse; in total we found eleven papers for the four challenge areas that were relevant to the company's situation. The organisation commented on the usefulness of our findings and reports an intention to adopt some suggestions.

\section{LESSONS LEARNED}

We are still in the early days of using this approach as we have only reached the end of the investigation stage with two projects. However, both have yielded valuable results and led to the integration of research and practice. Here we discuss some of the lessons learned.

Building trust: An advantage of our time-boxed approach is that we build trust with our collaborators by giving feedback early and frequently. One way we do this is by making regular visits during the investigation stage and giving verbal feedback about our ideas and our progress at each meeting. This helps us to check that our collaborators are happy with our progress. When we write papers, we always show our collaborators our work and encourage them to add to it. We find that getting regular feedback both for our research and writing helps to promote continuous improvement. We have noticed that change starts happening as soon as we engage with practitioners. The decision about which changes to make is left with the organisation, so the implementation cycle belongs more to the organisation than it does to us.

Appropriate contracts: We ensure we have written agreement at the beginning of the project before we start doing any work. We find this level of formality helps the smooth running of the project. As part of this we also sign a non-disclosure agreement. By doing this we are ensuring that we get buy-in from everyone who is likely to be involved. At the Kick-off stage we often talk to managers, and this is important as we need to get managerial approval. However, if we need to work with agile team members, we also need buy-in from those individuals. Although we always have one point of contact, we have learned that we need to set up a secondary contact, as in both of our initial projects we had problems when our contact was on holiday.

Flexibility: We have learned that we need to maintain a flexible approach as things change on agile projects. We have also found a full-time researcher essential so that we can maintain this flexibility. For example we often need to be very flexible about when we visit our host organisation, and need to be able to arrange a visit at short notice, or delay a visit depending on circumstances. Taking business priorities into consideration inevitably had consequences to our timeline. However, having an incremental model for delivering outputs allows us to capture what we have learned in a timely manner.

Outputs tailored to specific audiences: One of our aims has been to ensure that we produce accessible papers from our work that are tailored to different audiences. Our first output is always a white paper aimed at the practitioner community. This highlights the challenges found in the case study, summarises the relevant literature and identifies successful changes and areas of best practice. Our white papers are freely available on our website (www.agileresearchnetwork.org). By doing this we aim to show that research outputs can be made accessible, to show agile practitioners that they are not alone, and to enhance understanding of how agile methods are used in practice. In our academic papers we highlight the richness and complexity of investigating challenges faced in organisations.

Funding: The current funding model has run for one year, and has been renewed for another. We ask collaborators to pay for our travel, as we believe that paying for some part of the research helps to seal their commitment to its value. Future funding for ARN could continue with a similar consortium or membership model.

Research expertise: The two case studies so far have drawn on the research team's expertise. We would not be able to take on board any organisational challenge for which we did not already have some level of expertise. However, once we start our investigation we often find ourselves taking unexpected turns and this approach has been a learning experience for the team, and has widened our knowledge of the literature.

\section{CONCLUSION}

The two organisations studied so far are very different; they have provided different challenges requiring different data gathering methods and have produced different outcomes. Our model of engagement has proved to work effectively in these two cases, coping with a range of situations.

The outcomes produced from each challenge are dependent on the uniqueness of the context and level of available literature. For example, in the case of UX there was sufficient literature for us to synthesise and summarise for practitioner use. However, in the second case, there proved to be little existing literature relevant to our specific case, leading to the identification of an underresearched area adding to the research agenda in this area.

We acknowledge that the timelines of our model are aspirational, even though they are elapsed time rather than calendar time. We will review them for the next evolution of our model.

\section{ACKNOWLEDGMENTS}

Our thanks to our collaborators and the DSDM Consortium.

\section{REFERENCES}

[1] DSDM Consortium. www.dsdm.org - accessed 31-01-2014

[2] Highsmith (2002) Agile software development ecosystems, Addison Wesley Professional

[3] Rosemann, M., \& Vessey, I. (2008). Toward improving the relevance of information systems research to practice: the role of applicability checks. MIS Quarterly, 32(1), 1-22 